\title{
Effect of Secondary transmission on Primary Pilot Carriers in Overlay Cognitive Radios
}

\author{
Yihenew Beyene, Kalle Ruttik and Riku Jäntti \\ Department of Communications and Networking \\ Aalto University, School of Electrical Engineering \\ Espoo, Finland \\ yihenew.beyene@aalto.fi, kalle.ruttik@aalto.fi, riku.jantti@aalto.fi
}

\begin{abstract}
Cognitive radio aims to increase spectral efficiency by exploiting information about the environment and activity of other transmitters. It allows access to temporary and/or spatially unused TV spectrum, white space, or overlay on the TV transmission. In this paper we consider an overlay cognitive radio in DVB-T coverage area, TV black space. We analyze the performance of a simple sequential interference cancellation algorithm at secondary receiver in presence of channel estimation error. The channel estimation quality depends on how the overlay signal disturbs the primary pilots. We compare two OrthogonalFrequency Division Multiplexing based overlay cognitive radio schemes having secondary with: 1) modified WLAN frame structure avoiding secondary transmission on primary pilots. 2) LTE-type frame structure that uses all the DVB-T carriers. A prototype system was implemented on hardware platform to validate analytical results and study implementation limitations. Simulation and measurement results show that overlay interference on primary pilots does not have significant impact on primary reception. However, unlike scheme (1), in scheme (2) signal-to-interference plus noise ratio at secondary receiver was found to be severely limited by interference level on pilot carriers.
\end{abstract}

\section{INTRODUCTION}

Cognitive radio can be employed to increase spectral efficiency by allowing flexible sharing of the radio resource among multiple users. Based on the spectrum occupancy and available information to the cognitive transmitter, a cognitive radio system may opportunistically transmit on unoccupied spectrum, white space, or overlay its signal on top of other transmission. In this paper we analyze how an overlay cognitive radio can coexist with DVB-T broadcasting system.

A cognitive radio research has extensively covered question of how to use TV white spaces. Unfortunately, TV white space is available mostly in areas with low population density [1]. The large customer base can be targeted better if the secondary system operates in TV networks coverage areas, in TV black space. A key for unlocking TV black space is provided by the overlay type cognitive radio [2].

The overlay transmitter is an cognitive system that uses knowledge of primary signal for combing its own signal with primary transmission. In this paper we consider that the cognitive system has the knowledge of the primary signal and concentrate our attention on the performance of secondary overlay receiver.

We consider the type of overlay transmission that hides itself from primary receivers. This special case is interesting since the equipments of incumbent users do not need any modifications. All the systems coexistence related complexity is handled by the secondary system. The secondary system relays the primary signal to compensate for the interference it causes to the primary receiver.

The overlay secondary receiver can be described as well as the well known interference cancellation (IC) problem. In order to decode its own signal the secondary receiver has first to remove the strong TV signal. It decodes the TV signal and subtracts it from the received signal. The residual signal, ideally, contains only secondary signal and noise. This kind of sequential interference cancellation allows for interferencefree communication when the interference is very strong and channel is known [3]. The problem arises when the channel estimation error does not allow to compensate the whole TV signal. A practical TV system requires that the overlay secondary signal is about $20 \mathrm{~dB}$ weaker than the TV signal. The residual error after the IC step could easily be in the same order and the secondary signal will have very low signal-tointerference plus noise ratio (SINR) level.

The channel estimation quality depends on SINR level of the pilot signal. In many popular commercial multi-carrier communication systems, globally known pilots symbols are transmitted periodically on chosen set of carriers. By computing the channel response on these carriers, the receiver applies predictive or interpolation techniques to estimate the channel coefficients on other adjacent carriers. The channel estimation quality depends on how the overlay secondary system spreads its signal. When there is overlay transmission on pilot carriers, channel estimation error will increase.

In this paper we present two alternative overlay approaches: one where the overlay system avoids interfering the primary system pilot symbols and the other where the secondary signal is overlaid over all the primary symbols. We illustrate the analysis by considering two practical secondary systems one of them has modified WLAN type frame structure and the other has LTE-type frame structure. These two approaches emerged from analysis of the candidate secondary overlay systems. A WLAN (IEEE 802.11) type frame contains one Orthogonal-Frequency Division Multiplexing (OFDM) pilot symbol (preamble) followed by multiple data symbols. It is relatively easy to modify the frame structure of the preamble such that its carriers are orthogonal with primary DVB-T 
pilots. On the other hand, the LTE frame contains scattered pilot locations [4], and it is more difficult to modify this structure.

It is claimed that the overlay system suffers from multiple practical implementation related errors [5]. Wide variety of error sources make the error analysis to be very challenging exercise. In the paper we make detailed analysis of only one type of error: channel estimation related error. In order to consider the contribution of other type of errors we made a prototype of the system by using a universal software radio peripheral (USRP) hardware platform [6]. We validate our analytical results with simulations and also by measurements from this prototype hardware implementation.

\section{SYSTEM MODEL}

We consider a secondary system that overlays its signal over primary DVB-T broadcasting signal. The same conclusion can be derived for any primary broadcasting system where secondary systems can get access to the broadcast message. The overlay system is designed such that the TV receivers can continue to operate unchanged. This is achieved by relaying primary signal from the secondary receiver in order to compensate for the interference.

We model the overlay system as two-input-two-output interference channel with asymmetric side information [7]. We denote one transmitter receiver pairs as primary system link, indexed by $p$, and the secondary system link, indexed by $s$, see Fig. 1. Accordingly to the overlay approach [7], the secondary transmitter dedicates fraction $\alpha$ of its transmission power $P_{s}$ for improving the primary signal level. It knows the primary system signal $X_{p}$ and combines the primary signal with its own information signal $X_{s}$. Outputs of the overlay channel are given by

$Y_{p}=h_{p p} \sqrt{P_{p}} X_{p}+h_{s p}\left[\sqrt{\alpha P_{s}} X_{p}+\sqrt{(1-\alpha) P_{s}} X_{s}\right]+Z_{p}$

$Y_{s}=h_{p s} \sqrt{P_{p}} X_{p}+h_{s s}\left[\sqrt{\alpha P_{s}} X_{p}+\sqrt{(1-\alpha) P_{s}} X_{s}\right]+Z_{p}$

where received signals contain transmitted information $X_{p}$, $X_{s}$ and noise term $Z_{p}, Z_{s}$. The channel is described by transmission powers $\left(P_{p}, P_{s}\right)$ and amplitude of channel coefficients $\left(h_{p p}, h_{s p}, h_{p s}, h_{s s}\right)$. In the channel model the first index indicates the transmitter type and the second index indicates the receiver type. The signal and noise components are modeled as complex Gaussian random variables with average signal powers normalized to $1, E\left[\left|X_{p}\right|^{2}\right]=E\left[\left|X_{s}\right|^{2}\right]=1$, and noise powers $N_{p}, N_{s}$ respectively.

We assume that the primary system is TV transmission that has to be supported in certain coverage area. The TV transmission is characterized by a fixed required data rate $R_{p}$. By assuming that the transmitted signals appear Gaussian the data rate is limited by the Shannon capacity equation

$$
R_{p} \leq C_{1}(\alpha)=\log \left[1+\frac{\left|h_{p p}\right|^{2} P_{p}+\left|h_{s p}\right|^{2} \alpha P_{s}}{N_{p}+\left|h_{s p}\right|^{2}(1-\alpha) P_{s}}\right]
$$

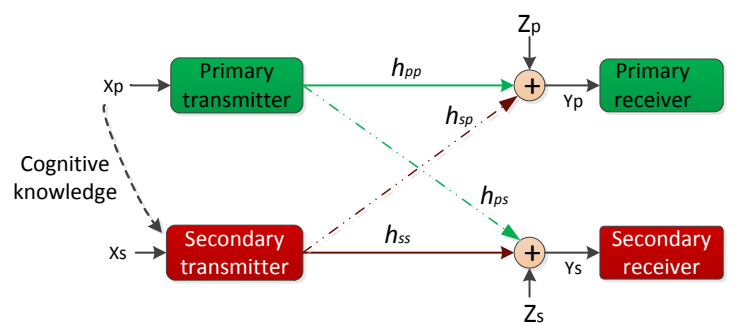

Fig. 1: A two-input-two-output cognitive radio channel with the secondary transmitter having non-causal knowledge of the primary signals.

where the total signal power is computed as non coherent combination of signals from primary and secondary transmitters.

The fraction $\alpha$ is function of channel coefficients. Each location of primary receiver requires different $\alpha$ value. In practice, we set $\alpha$ to satisfy the TV reception in the full TV coverage area. Such guarantee can be given if we set $\alpha$ to satisfy the TV reception even for very weak primary transmitter signal levels at the cell edges. In the extreme case we could ignore the primary transmitter's signal all together and approximate the received signals as

$$
\begin{aligned}
& Y_{p_{\text {worst }}}=h_{s p}\left(\sqrt{\alpha P_{s}} X_{p}+\sqrt{(1-\alpha) P_{s}} X_{s}\right)+Z_{p} \\
& Y_{s_{\text {worst }}}=h_{s s}\left(\sqrt{\alpha P_{s}} X_{p}+\sqrt{(1-\alpha) P_{s}} X_{s}\right)+Z_{s}
\end{aligned}
$$

where $\alpha$ is chosen to meet the minimum SINR required by the primary receiver. This ensures unaltered operation of the primary receiver. In the rest of this paper, we assume such worst case scenario for validating performance of the overlay system.

Now, we focus on performance of overlay receiver which sees strong interference from primary TV signal. Since the interference power is relatively high, the receiver can achieve acceptable performance by using a simple interference cancellation algorithm. It first estimates and removes the primary signal and then proceeds with decoding of its own signal. The distinctive feature of our setup is that the secondary signal has very low level. In this case sequential decoding is impaired by the channel estimation error during decoding of the primary signal. This error is not only a function of noise but depends also on how the overlay system allocates its own signal.

Many latest wireless technologies such as WLAN, LTE and DVB-T are multi-carrier systems (OFDM based) where a certain set of carriers are used for transmitting globally known training symbols. The channel estimation quality depends on the SINR level on those training symbols. In the next section we describe the structure of training symbols in DVB signal and model how the overlay transmission impacts the quality of channel estimation and due that the performance of the interference cancellation scheme.

\section{A. Pilots structure in DVB-T signal}

In a DVB-T physical layer each of the transmitted OFDM symbols carries one MPEG-2 transport stream packet. Two 


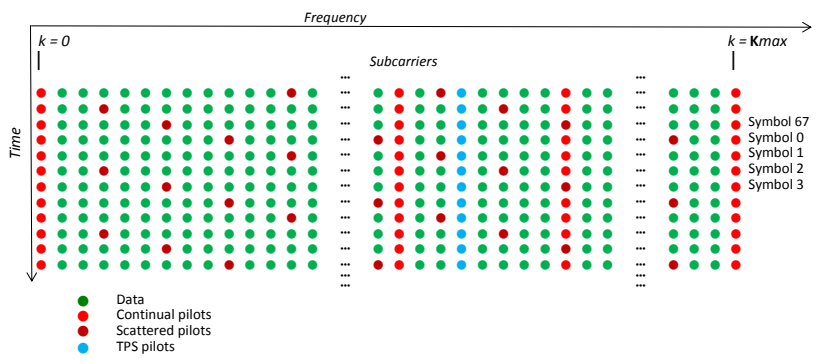

Fig. 2: Illustration of DVB signal pilot structure.

types of reference pilots for channel estimation are transmitted over a set of OFDM carriers. Location of first set of pilot signals, called continual pilots, is fixed while scattered pilots [8] change their location from symbol to symbol as shown in Fig. 2. In $8 \mathrm{~K}$ mode ( $8 \mathrm{MHz}$ bandwidth) there are 6817 carriers among which 177 carriers are continual pilots. In every 12 carriers one is assigned for scattered pilots.

\section{B. Secondary system}

In sequential decoding scheme, the receiver estimates, reconstructs and subtracts the primary signal from the received signal. The secondary signal is then decoded from the residual signal. From Equation (5), the received signal at the secondary receiver can be re-written as

$$
\begin{aligned}
Y_{s} & =h_{s s} \sqrt{(1-\alpha) P_{s}} X_{s}+h_{s s} \sqrt{\alpha P_{s}} X_{p}+Z_{s} \\
& =h_{s s} \sqrt{(1-\alpha) P_{s}} X_{s}+H X_{p}+Z_{s}
\end{aligned}
$$

where $H=h_{s s} \sqrt{\alpha P_{s}}$.

After compensation, the residual signal can be expressed as $Y_{R}=\sqrt{(1-\alpha) P_{s}} h_{s s} X_{s}+(H-\hat{H}) X_{p}+\hat{H}\left(X_{p}-\hat{X}_{p}\right)+Z_{s}$

where $\hat{H}$ is the channel estimate, $H-\hat{H}$ is the channel estimation error, and $\hat{X}_{p}$ is the estimate of the transmitted primary symbols.

The overlay secondary receivers are located in the same coverage area as the primary users. In the coverage area the primary signal has high SINR level. Because of that, the symbol estimation error, $X_{p}-\hat{X}_{p}$, is relatively small and its impact on the secondary reception can be ignored. The $\operatorname{SINR}_{s}$ after interference cancellation is

$$
\begin{aligned}
\operatorname{SINR}_{s} & =\frac{\left|h_{s s}\right|^{2}(1-\alpha) P_{s}}{|H-\hat{H}|^{2} P_{p}+N_{s}} \\
& =\frac{1 / \gamma}{\left(\left|h_{s s}-\hat{h}_{s s}\right|^{2}\right) /\left|h_{s s}\right|^{2}+1 / \mathrm{SNR}_{p}}
\end{aligned}
$$

where $\mathrm{SNR}_{p}=\frac{\left|h_{s s}\right|^{2} \alpha P_{s}}{N_{s}}$ is the signal-to-noise ratio (SNR) of the primary signal, and $\gamma=\alpha /(1-\alpha)$ is primary to secondary signal power ratio.

The secondary signal quality is inversely proportional to normalized variance of the channel estimation error, $\mid h_{s s}-$ $\left.\left.\hat{h}_{s s}\right|^{2}\right) /\left|h_{s s}\right|^{2}$. The quality of the channel estimation depends on the $\operatorname{SINR}_{p}$ level of the primary pilots. The overlay system can choose how much interference it generates to the primary pilot symbols. We quantify how much the performance could be improved if the overlay transmission would avoid transmission on primary signal pilot carriers. We illustrate the analysis with two extreme cases: interference-free pilot (IFF) and interfered pilot (IFR). Performance estimation in these two cases is made for a flat block-fading channel.

In the following subsections we will derive maximum achievable SNR of the secondary signal for the above two scenarios using the Cramér-Rao Lower Bound (CRLB) of channel estimation error.

\section{Interference-free pilot (IFF)}

In this scenario, the secondary transmission is orthogonal to the primary pilot carriers. As the pilot carriers are free from secondary interference, signal received by the secondary receiver on these carriers is expressed as

$$
Y_{s}^{p}=h_{s s} \sqrt{\alpha P_{s}} X_{p}+Z_{s} .
$$

Assuming $h_{s s}$ and $X_{p}$ to be independent, the conditional probability of received signal is given by

$$
f\left(Y_{s}^{p} \mid h_{s s}, X_{p}\right)=\frac{1}{\pi N_{s}} e^{-\left|Y_{s}^{p}-h_{s s} \sqrt{\alpha P_{s}} X_{p}\right|^{2} / N_{s}} .
$$

Usually, the channel estimator uses multiple pilot carriers. Let pilot carriers, $\mathbf{X}_{p}=\left\{X_{p}[1], X_{p}[2], \cdots, X_{p}[N]\right\}$ be used for estimating the single-tap (flat fading) channel. The joint probability density function is

$$
f\left(\mathbf{Y}_{s}^{p} \mid h_{s s}, \mathbf{X}_{p}\right)=\prod_{n=1}^{N} \frac{1}{\pi N_{s}} e^{\left.-\mid Y_{s}^{p}[n]-h_{s s} \sqrt{\alpha P_{s}} X_{p}^{[} n\right]\left.\right|^{2} / N_{s}} .
$$

We derive the CRLB from derivative of the likelihood function

$$
\begin{aligned}
\mathcal{L}\left(\mathbf{Y}_{s}^{p} \mid h_{s s}, \mathbf{X}_{p}\right) & =\log \left(f\left(\mathbf{Y}_{s}^{p} \mid h_{s s}, \mathbf{X}_{p}\right)\right) \\
\frac{\partial \mathcal{L}\left(\mathbf{Y}_{s}^{p} \mid h_{s s}, \mathbf{X}_{p}\right)}{\partial h_{s s}} & =I\left(h_{s s}\right)^{*}\left(\hat{h}_{s s}-h_{s s}\right)^{*}
\end{aligned}
$$

where $(\cdot)^{*}$ stands for the complex conjugate and

$$
\hat{h}_{s s}=\frac{\sum_{n=1}^{N} Y_{s}^{p}[n] X_{p}^{*}[n]}{\sqrt{\alpha P_{s}} \sum_{n=1}^{N}\left|x_{p}[n]\right|^{2}}
$$

is the minimum-variance unbiased (MVU) estimator, and

$$
I\left(h_{s s}\right)=\frac{\alpha P_{s} \sum_{n=1}^{N}\left|x_{p}[n]\right|^{2}}{N_{s}}=\frac{\alpha N P_{s}}{N_{s}}
$$

is the Fisher information related to CRLB of variance of $\hat{h}_{s s}$ as $\mathrm{CRLB}=1 / I\left(h_{s s}\right)$.

Variance a MVU estimator, $\hat{h}_{s s}$, is the same as variance of the estimation error, $\tilde{h}_{\mathrm{IFF}}=h_{s s}-\hat{h}_{s s}$. Hence the minimum error variance is

$$
\min \left\{\operatorname{Var}\left[\tilde{h}_{\mathrm{IFF}}\right]\right\}=\frac{N_{s}}{N \alpha P_{s}}=\frac{\left|h_{s s}\right|^{2}}{N \cdot \mathrm{SNR}_{p}} .
$$

After combining (10)) and (18), the maximum secondary $\mathrm{SINR}_{s}$ in IFF scheme is

$$
\max \left\{\operatorname{SINR}_{\mathrm{IFF}}\right\}=\frac{\mathrm{SNR}_{p}}{\gamma(1+1 / N)} .
$$




\section{Interfered pilot (IFR)}

In IFR transmission the secondary transmitter overlays its signal over all OFDM carriers. Unlike IFF this approach allows to maximize the spectrum usage. However, the additional disturbance on primary pilots channel estimation quality reduces secondary signal $\mathrm{SINR}_{s}$ and data rate.

As there is secondary transmission on all carriers, received signal on a pilot carrier is expressed as

$$
Y_{s}^{p}=h_{s s} \sqrt{\alpha P_{s}} X_{p}+h_{s s} \sqrt{(1-\alpha) P_{s}} X_{s}+Z_{s} .
$$

A simple interference canceler treats the secondary signal $X_{s}$ as zero-mean complex Gaussian noise and we can rewrite Equation (20) as

$$
Y_{s}^{p}=h_{s s} \sqrt{\alpha P_{s}} X_{p}+Z^{\prime}
$$

where $Z^{\prime} \sim \mathcal{C N}\left(0, \sigma_{z^{\prime}}^{2}\right)$ and $\sigma_{z^{\prime}}^{2}=N_{s}+\left|h_{s s}\right|^{2}(1-\alpha) P_{s}$.

By following the same steps as in case of IFF we get

$$
\begin{aligned}
\hat{h}_{s s} & =\frac{\sum_{n=1}^{N} Y_{s}^{p}[n] X_{p}^{*}[n]}{\sqrt{\alpha P_{s}} \sum_{n=1}^{N}\left|x_{p}[n]\right|^{2}} \\
I\left(h_{s s}\right) & =\frac{\alpha P_{s} \sum_{n=1}^{N}\left|x_{p}[n]\right|^{2}}{\sigma_{z^{\prime}}^{2}}=\frac{\alpha N P_{s}}{N_{s}+\left|h_{s s}\right|^{2}(1-\alpha) P_{s}}
\end{aligned}
$$

Compared to the IFF scenario the estimator in IFR case has higher variance (CRLB). Minimum of variance of the estimation error, $\tilde{h}_{\mathrm{IFR}}=h_{s s}-\hat{h}_{s s}$ is

$$
\begin{aligned}
\min \left\{\operatorname{Var}\left[\tilde{h}_{\mathrm{IFR}}\right]\right\} & =\frac{N_{s}+\left|h_{s s}\right|^{2}(1-\alpha) P_{s}}{\alpha N P_{s}} \\
& =\frac{1}{N}\left(\frac{\left|h_{s s}\right|^{2}}{\operatorname{SNR}_{p}}+\frac{\left|h_{s s}\right|^{2}}{\gamma}\right) .
\end{aligned}
$$

Combining Equations (10) and (25), the maximum IFR secondary SINR is obtained as

$$
\max \left\{\operatorname{SINR}_{\mathrm{IFR}}\right\}=\frac{\mathrm{SNR}_{p}}{\gamma(1+1 / N)+\mathrm{SNR}_{p} / N} .
$$

\section{NUMERICAL ILLUSTRATIONS}

We illustrate our results by simulating the performance of secondary system. We also validated the results by prototyping the system in a real hardware platform. The prototype system is implemented by using PC and USRP based software radio platform [6].

In our simulations the primary TV system uses DVB-T physical layer without channel coding. The secondary system overlays its own OFDM signal on the DVB-T OFDM frame structure. The secondary system is synchronized to DVB-T frames and it knows the location of the primary system pilots. A DVB-T system contains both continual pilots and scattered pilots. For simplifying the implementation we consider only the continual pilot locations in our simulation and prototype platform.

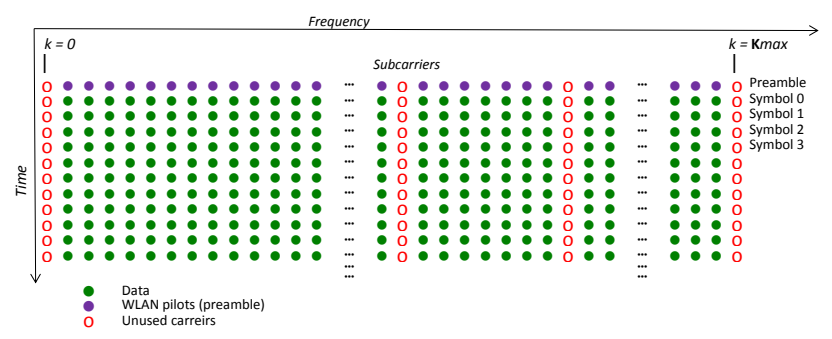

Fig. 3: Proposed frame structure for IFF overlay transmitter.

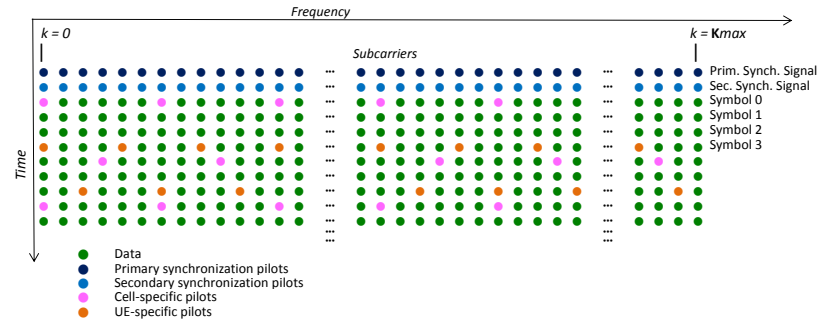

Fig. 4: Proposed frame structure for IFR overlay transmitter with synchronization symbols and reference pilots.

In IFF case the secondary system uses packet based transmission (see Fig. 3). Primary and secondary system OFDM symbols are time synchronized and have the same amount of OFDM carriers as the primary symbols. The secondary packets are generated at random instances and can span multiple subsequent OFDM symbols. The secondary system packet structure resembles the WLAN physical layer where known pilot sequence, preamble, is transmitted at the beginning of each packets. The preamble is used for synchronization and channel estimation of secondary signal. The secondary transmission is made in such a way that in all OFDM symbols including the preamble, no transmission occurs on the DVB continual pilot carriers.

In IFR overlay transmission, the secondary transmitter uses all carriers. In this case we implement the secondary system to use LTE frame structure (Fig. 4). The physical layer frame is divided into subframes each of which contains 14 OFDM symbols. Pilot signals which are used for secondary channel estimation are transmitted on defined set of carriers. These pilots are distributed in time and frequency, and hence their location varies from one OFDM symbol to another.

In Fig. 5 we illustrate how well a CRLB describes the channel estimation quality. In Fig. 5 we plot the secondary signal SINR when the primary signal SNR is treated as the argument and the plotted curves are parametrized with target $\mathrm{SINR}_{p}$ values. As predicted by (25) in IFR at high SNR level the interference in pilots dominates the estimation quality. At high SNR the noise impact is very small and SINR is leveled off at given interference level. In IFF system the pilots are interference free and such limits does not exists.

The secondary system reception quality is the function of the primary signal target SINR level and the noise level in the system. Calibrating noise level of the USRP was difficult due to variability of internal noise for different power levels. We 


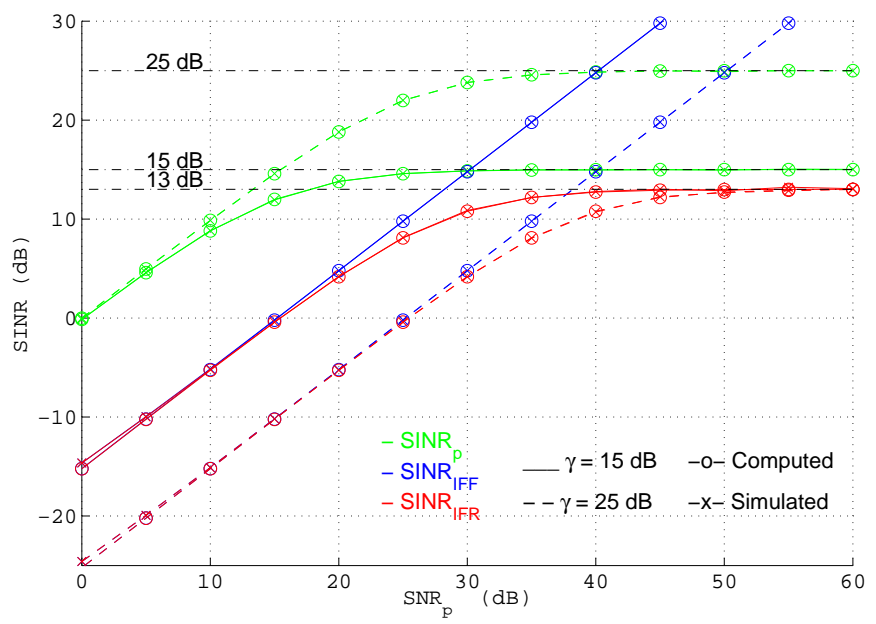

Fig. 5: IFF and IFR received SINR, $N=20$.

set the transmit power large enough and expose the receiver to controlled white Gaussian noise which is sufficiently higher than receiver internal noise. Received signal at the secondary receiver is given by

$Y_{s s}=h_{s s} \sqrt{\alpha P_{s}} X_{p}+h_{s s} \sqrt{(1-\alpha) P_{s}} X_{s}+Z_{c}+Z_{u}$

where $Z_{u}$ is USRP's internal noise, and $Z_{c} \sim \mathcal{C N}\left(0, N_{c}\right)$ is controlled external noise with $N_{c}>E\left\{\left|Z_{u}\right|^{2}\right\}$. We approximate primary and secondary SNR as

$$
\begin{aligned}
& \mathrm{SNR}_{p} \approx \frac{E\left\{\left|h_{s s} \sqrt{\alpha P_{s}} X_{p}\right|^{2}\right\}}{E\left\{\left|Z_{c}\right|^{2}\right\}}=\frac{\alpha\left|h_{s s}\right|^{2} P_{s}}{N_{c}} \\
& \mathrm{SNR}_{s} \approx \frac{E\left\{\left|h_{s s} \sqrt{(1-\alpha) P_{s}} X_{s}\right|^{2}\right\}}{E\left\{\left|Z_{c}\right|^{2}\right\}}=\frac{(1-\alpha)\left|h_{s s}\right|^{2} P_{s}}{N_{c}} .
\end{aligned}
$$

This approximation is valid only for low SNR targets due to power headroom limitation of the hardware. Practical primary protection constraint requires $\gamma>10 \mathrm{~dB}$ or $\alpha>0.9$ which leaves the secondary signal with small fraction of the total power. Therefore, it is difficult to reach high SNR for secondary user as at high SNR the hardware internal noise becomes the dominating noise component.

In our illustrations in Fig. 6 and 7 we have fixed the target Signal-to-interference ratio (SIR) level between the primary and secondary signal levels, $\gamma=\alpha /(1-\alpha)$, and evaluate the receiver's performance for different SNR levels. Fig. 6 and 7 contain comparisons between the simulated results and measurements from hardware platform implementation.

It can bee seen that primary system's performance has not been degraded due to secondary transmissions on its pilot carriers. With respect to the primary signal level the secondary signal level is very low and such small interference does not impact the primary connection.

The secondary signal's BER in IFF scheme is sufficiently close to the theoretical performance except that it has been bounded by the primary signals BER especially at higher SNR. At high SNR we see a big discrepancy between simulated and measured results. The internal noise level of our hardware

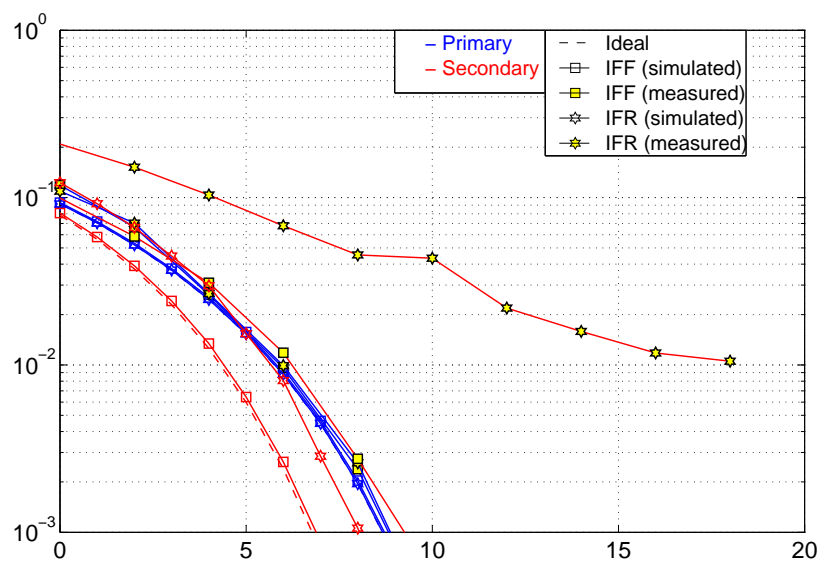

(a) Primary: 4QAM, Secondary: 4QAM

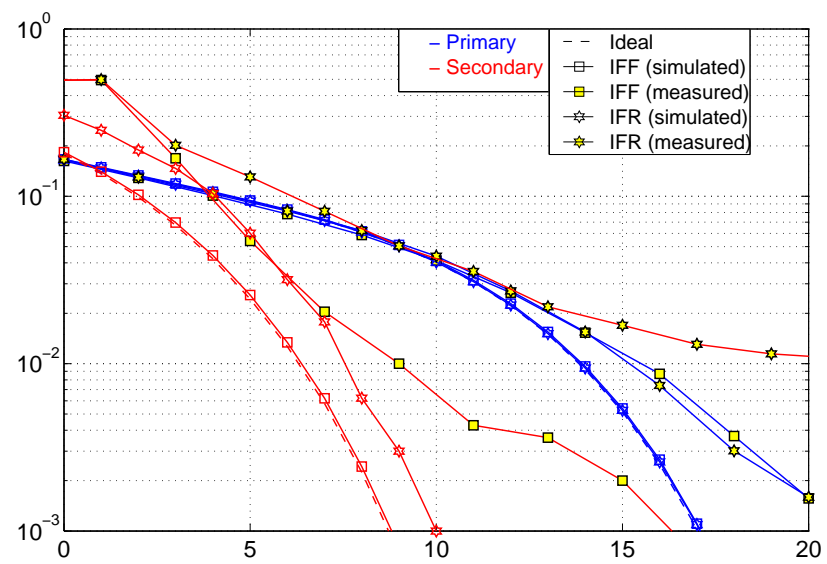

(b) Primary 16QAM, Secondary: 4QAM

Fig. 6: Measured BER for primary and secondary signals, $\gamma_{p}=12 d B$.

platform is in order of $20 \mathrm{~dB}$. The hardware internal noise is an additional disturbance that is not included into analytical model and simulations but it is dominating the performance of the implemented prototype system.

We considered also overlay system performance with different primary system modulation schemes. In higher modulation schemes, where euclidean distance between constellation points is smaller, we can allow only very low secondary signal's power levels. As can be seen from Fig. 7a and 7b, higher $\gamma_{p}$ value gives better performance. One can observe that reducing secondary signal power relative to the primary signal (increasing $\gamma_{p}$ ) also improves the secondary receiver's performance.

\section{CONCLUSION}

We have quantified the impact the overlay transmission has on the interference cancellation in secondary receiver. The question we analyzed is whether the overlay secondary system should allocate its own signal on primary pilots or not. We consider a primary system that uses DVB-T transmission and the secondary overlay system does not change the SINR of the primary signal. The secondary signal level is very low. Even 


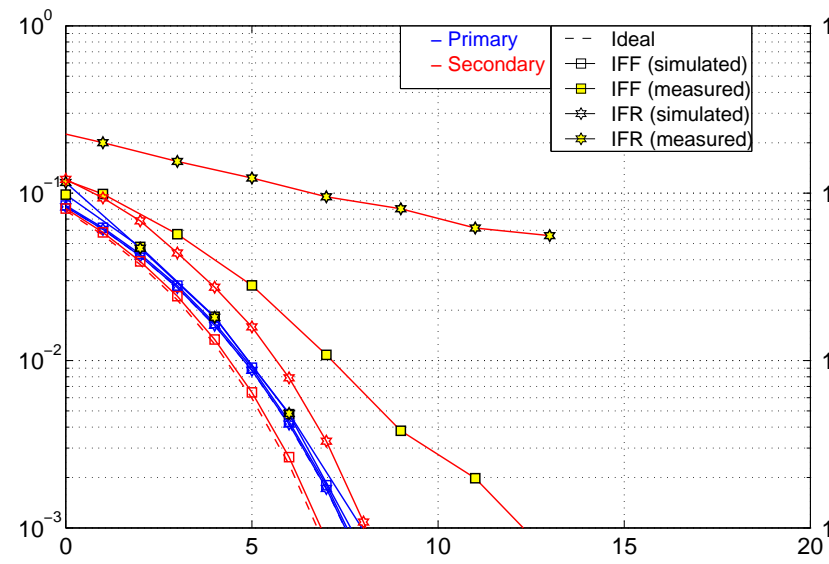

(a) Primary: 4QAM, Secondary: 4QAM

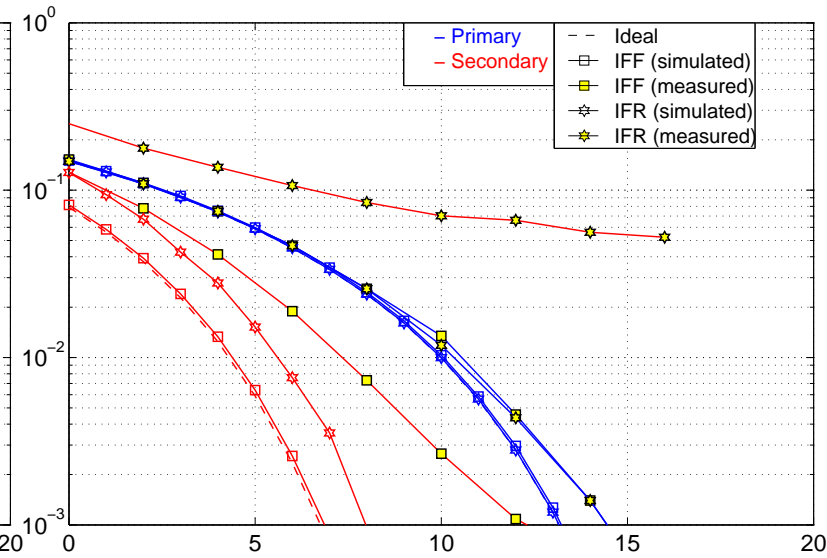

(b) Primary 16QAM, Secondary: 4QAM

Fig. 7: Measured BER for primary and secondary signals, $\gamma_{p}=17 d B$.

when we allocated the secondary signal on primary pilots the increase of estimation error due to the secondary interference is negligible from the primary signal point of view. However this small channel estimation error is in the same order as the secondary signal level. Therefore it is preferable for overlay system not allocate its own signal on primary pilots. The small interference increase does not impact primary receivers but deteriorates the secondary signal reception significantly.

We validated our analytical results by simulations and implementation of the overlay system in USRP hardware platform. Practical implementation of prototype overlay system using real USRP platform confirms analytical results. However, due to quantization error and phase noise of the hardware, measurement results start to diverge from the simulation results at higher SNR values. We can observe how at the high SNR levels the hardware inherent distortions start to dominate the error. However, it is encouraging to see that even on this low budget hardware platform hardware limitation can be ignored as long as SNR on secondary channel is less than $20 \mathrm{~dB}$.

\section{REFERENCES}

[1] J. van de Beek, J. Riihijärvi, and A. M. Achtzehn, "UHF white space in Europe - a quantitative study into the potential of the $470-790 \mathrm{MHz}$ band," in IEEE Symposium on In New Frontiers in Dynamic Spectrum Access Networks (DySPAN), 2011, pp. 178-188.

[2] A. Goldsmith, S. Jafar, I. Maric, and S. Srinivasa, "Breaking spectrum gridlock with cognitive radios: An information theoretic perspective," Proceedings of the IEEE, vol. 97, no. 5, pp. 894 -914, may 2009.

[3] A. Carleial, "Interference channels," Information Theory, IEEE Transactions on, vol. 24, no. 1, pp. 60 - 70, jan 1978.

[4] “3GPP TS 36.211 v8.9.0 - $3^{\text {rd }}$ Generation Partnership Project; Technical Specification Group Radio Access Network; Evolved Universal Terrestrial Radio Access (E-UTRA); physical channels and modulation (Release 8)," Tech. Rep., 2009.

[5] Y. Selen, R. Baldemair, and J. Sachs, "A short feasibility study of a cognitive TV black space system," in Personal Indoor and Mobile Radio Communications (PIMRC), 2011 IEEE 22nd International Symposium on, sept. 2011, pp. $520-524$.

[6] (2011, December 25) Ettus research 1lc. [Online]. Available: http://ettus.com/products

[7] A. Jovicic and P. Viswanath, "Cognitive radio: An information-theoretic perspective," in Information Theory, 2006 IEEE International Symposium on, july 2006, pp. $2413-2417$.

[8] EN 300744 V1.6.1, "European Standard (Telecommunications series) Digital Video Broadcasting (DVB); Framing structure, channel coding and modulation for digital terrestrial television," STSI, Tech. Rep., 2009. 